\title{
Resultados alejados de la atención hospitalaria infantil
}

\author{
Ana M. Kaempffer R.' $;$ Emesto Medina L. '; Edith Comejo A. ${ }^{2}$; Enrique Hernandez A. ${ }^{3}$
}

\begin{abstract}
Resumen
Objetivo: estajlecer los resuliados alejcdos de la hospitalización explorando la condición de los egresados 30 , 180 y 365 días después del alta, sepcrando los grupos de sanos y recuperados, problemos persistentes, empeoromiento, rehospilalización y fallecimiento. Pocientes y métodos: se estudió ura muesira alealoría de 1 Do entermos ingresados en 1997 a los hospitales San José, Roberto del Rio y Clinica de la Universidad de Chile. Un total de 414 cosos corresponden a niños y adolescentes que constiluyen el motivo de esia comunicación. Los pacientes fueron visitados en sus domicjlios, identificandose su condición alejada, el cumplimiento de las indicaciones posaila, como asimismo la magnilud del gaslo en alencion médico y opiniones sobre la alención recibida. Resulfados: el $70 \%$ de los cosos se encontrabon sanos y recuperados 30 días después del alta, subiendo o 75,9\% a los 6 meses. En 18,8\% se mantenía una condición alterado estable, $0,7 \%$ habian empeorodo, $9,5 \%$ necesitó una nueva hospilalización y $0,2 \%$ habion faltecido en el mes siguiente ol alta. Los resuliados son similares en ambos sexos. Difieren significativamente [p 0,051 de acuerdo o la edad con los mejores resultados en reciên nacidos y adolescentes de 15 a 19 años. La mayor proporción de recuperación correspondió a hospitalizaciones por enfermedades respiralorias e infectiosas trasmistbles. Una eslado hospitalaria entre 2 a 5 dias se asocia a mejores resultados. Se registran diferencics significalivas según tipo de trotamiento, condición al alla, cumplimiento de indicaciones posalta y tipo de previsión en solud. Conctusión: la atención hospilalosio infontil tiene un resuitado salisfoclorio, superior al registrado en adultos. con una baja letalicad posalta, pero con una alta tasa de rehospitalización.
\end{abstract}

(Polabros clave: hospital, pediático, hospitalización, alta.'?

\section{Children's hospital care: a follow-up after discharge}

Objective: to know the resulis of hospital care through a follow-up pertormed 30,180 and 305 ofter discharge. Method: an aleatory sample or 1000 potients assisled in 1997 from the public hospitals of the Northern area of Santiago were studied. Four huncred and fourleen of them were children or cdolescents and they were visiled af their homes. The survey identified the long term health condition, accomplishment of medicai indications after discharge. cosis of medical core and oppinions about the hospital core. Resutts: a $70 \%$ out of rutal coses were healihy and recovered one month after discharge; the :atio was $75,6 \%$ monl's afterwards. Stable condilion was present in $19 \%$. $1 \%$ were in a worse condilion and $9.5 \%$ required a new hospital admission. The ccse fatality rale dusing the first month affer discharge was 0,2\%. Results are similar in soth sexes. Best results were noticed in newioorn and adelescents 15-?9 years of age; in respiratory and communicable disease patients and hospital slays from 2-5 days. Significant differences were observed according to mair lype of trealment, condition at the moment of discharge, accomplishment or post discharge indications ond type of health insurance. Conclusions: children and adolescents hospiral care shows good results afler discharge, beter than adults's results, with a low farality rate, but a rather high need of a second hospitalization.

(Koy words: hospital, pediatrics, hospitalizotion, haspital discharge.]

I. Escuela de Salud Páblica, Facultad de Medicina, Uni. versidad de Chile.

2. Enfernera Universitaria. Escuela de Salud Pública, Fâcultad de Medicina, Universidad de Chile.

3. Profesor de Estadística Escuela de Salud Pútlica, Facultad de Medicina, Universidad de Chile.

Este trabajo forma parte del Proyecto de Investigación: Atención Médica: Evaluación de resultados en el corto y mediano plazo. Proyecto FONDECYT N ${ }^{\circ}$ 1970249-1997.
Los hospitales constituyen un componente fundamental en la atención médica de una comunidad $^{1,2}$. En el caso chileno, una dotación de camas hospitalarias relativamente baja ( 3 por 1000 habitantes) permite virtualmente ingresar a la totalidad de las embarazadas chilenas para ser asistidas profesionalmente en el momento del parto; resuelve una importante proporción 
de las necesidades quirúrgicas del país y permite la atención de enfermos graves y complejos. Si bien no se sabe con entera exactitud, se estima que $70 \%$ del gasto en salud que realiza el país se canaliza a través de servicios hospitalarios ${ }^{3}$. De lo anterior se desprender necesidades de buen funcionamiento y el uso de técnicas de evaluación que permitan mejorar tanto el enfoque de acción como los resultados finales. La evaluación de los hospitales ha solido utilizar indicadores de recursos y de funcionamiento (número de egresos, promedio de estada, índice de ocupación, indices de producción, letalidad intrahospitalaria), como igualmente de calidad de la atención y de costos de operación $n^{4}$.

Sin embargo, en la literatura médica chilena se encuentran pocos ejemplos de evaluación de resultados en el mediano o largo plazo y de costo-efectividad de los establecimientos. Frente a ello hemos deseado explorar el resultado alejado de la acción hospitalaria y la relación costo-efectividad de las prestaciones. En este informe se comunican los resultados obtenidos en cuanto a resultados de la atención hospitalaria de niños y adolescentes en un sector de Santiago.

\section{Pacientes y métodos}

La investigación ha explorado la condición alejada de 1000 pacientes ambulatorios del sector norte de Santiago y de 1000 pacientes atendidos en los hospitales San José, Roberto del Río y Clínico de la Univerșidad de Chile en 1997. Los enfermos ingresaron al estudio en forma aleatoria y en proporcion similar al volumen de egresos de los establecimientos. Están siendo visitados en sus domicilios 30,180 y 365 días después del alta. En Jas $I 000$ hospitalizaciones registramos un total de 414 casos en niños y adolescentes menores de 20 años, de los cuales 297 fueron atendidos en el Hospital Roberto del Río, I01 en el Hospital San José y 16 en el Hospital Clinico de la Universidad de Chile. Proceden de servicios de neonatología, lactantes, infecciosos, aislamiento, medicina y cirugfa infantil y de adulios. Las visitas domiciliarias han permitido conocet la condición alejada de los pacientes. clasificóndoles en asíntomáticos con recuperación completa: persistencia de la patología básica con recuperación de alguna complicación; problema básico persislente; rehospitalización: empeorauniento y fallecimiento.

Las fichas clinicas han eniregado información rejativa a edad, sexo, diagnósticos de egreso, motivo de hospitalización, dís de estada, tratamiento recibido, complicaciones, condiciones al alta e indicaciones posalto. La visita domiciliaria ha permitido conocer el estado alejado de los pacientes, el cumplimiento de las indicaciones, como igualmente opiniones sobre la calidas percibido de la atención hospitalaria, sistema previsional y costos de atención para las personas.

En este informe no se incluyen los resultados relativos a los costos de hospitalización institucionales y de las personas, que serán motivo de otra comunicación.

Para agrupar los diagnósticos se ha usado la Clasificación Internacional de Enfermedades I0" Revisión". Para el análisis estadístico se ha empleado, de preferencia, el test de $\mathrm{Ji}^{2}$, estimándose como significativos valores de $\mathrm{p}$ inferiores a 0,05 .

\section{Resultados}

Resultados globales: treinta días después del alta, $70 \%$ de los pacientes se encuentra sano y recuperado, mientras en $18,8 \%$ persiste el problema básico y $9.4 \%$ ha requerido una rehospitalización. La mejor condición observada en las niñas no alcanza significado estadístico (tabla 1).

Resultados según edad: como se advierte en la tabla 2 , los resultados a $\mathbf{3 0}$ días difieren significativamente según la edad de los nin̆os y adolescentes $(p-0,05)$ observándose tasas mayores de recuperación en recién nacidos ingresados a servicios de neonatología у еп adolescentes mayores de 15 años.

Resultado según tipo de afecciones: los diferentes tipos de problemas que generan necesidades de hospitalización infantil varían significativamente $(p-0.02)$ en cuanto a resultados un mes después del alta (tabla 3 ).

Resultados según duración de la estada hospitalaria: estas relaciones son complejas. Por una parte, una estada demasiado corta puede influir en el resultado alejado pero, por otra, una estada larga puede ser más bien la consecuencia de un proceso patologico complejo o difícil. La tabla 4 muestra las diferencias de resultados de acuerdo a la longitud de la estada, diferencias que alcanzan significado estadístico $(p-0,01)$.

Los resultados a un mes plazo, de hospitalizaciones muy breves ( 1 día), son significativamente inferiores a los obtenidos con hospitalizaciones entre 2 y 5 días. Las hospitalizaciones prolongadas (15 dias y más) corresponden probablemente a casos complejos, dada la alta frecuencia en la que se requirió una hospitalización.

Resultados según otras variables: en la tabla 5 aparecen los resultados de acuerdo a diversos tipos de factores. Todas las diferencias tienen significado estadístico $(\mathrm{p}-0,01)$. 
Tabla 1

Resultados a 30 días de niños y adolescentes hospitalizados

\begin{tabular}{|c|c|c|c|c|c|c|c|c|}
\hline Género & n casos & A & B & R e s u & $\begin{array}{l}\text { o s }(\%) \\
\text { D }\end{array}$ & $\mathbf{E}$ & $\mathbf{F}$ & Total \\
\hline Total & 414 & 70.0 & 0.7 & 18,8 & 9,5 & 0.7 & 0.2 & 100 \\
\hline Hombres & 226 & 66.8 & 0.9 & 21,7 & 9,3 & 0.9 & 0,4 & 100 \\
\hline Mujeres & 188 & 74,0 & 0,5 & 15,4 & 9.6 & 0,5 & - & 100 \\
\hline
\end{tabular}

Diferencia enlre géneros $p=+0,05$

A: Totalmente recuperados

B: Recuperados de una complicación de un problema bósico que persiste

C: Persistencia de la patología que motivó la hospitalización

D: Rehospitalizados

E: Empeorados

F: Fallecidos

Tabla 2

Resultados a 30 días según edad de niños y adolescentes

\begin{tabular}{|c|c|c|c|c|c|c|c|c|}
\hline Género & n casos & A & B & $\underset{\mathrm{C}}{R}$ e s u & $\begin{array}{l}0 \mathrm{~s} \\
\mathrm{D}\end{array}$ & $\mathbf{E}$ & $\mathbf{F}$ & Total \\
\hline-1 mes & 106 & 84.0 & 0,9 & 3.8 & 11,3 & - & - & 100 \\
\hline $\ln 5 \mathrm{~m}$ & 56 & 60,7 & - & 21.4 & 14,3 & 1,8 & 1,8 & 100 \\
\hline 6 a $11 \mathrm{in}$ & $4 !$ & 68,3 & - & 19,5 & 12,2 & - & - & 100 \\
\hline $\mathrm{la} 4 \mathrm{a}$ & 90 & 73,4 & 2,2 & 14.4 & 7.8 & 2,2 & - & 100 \\
\hline 5 a $9 a$ & 58 & $5 B, 6$ & - & 37,9 & 3,4 & - & - & 100 \\
\hline $10 a 14 a$ & 43 & 58.1 & - & 32,6 & 9,3 & - & - & 100 \\
\hline Is a 19 a & 15 & 86.6 & - & 6,7 & 6,7 & - & - & 100 \\
\hline Total & 409 & 70,0 & 0,7 & 18,8 & 9.5 & 0,7 & 0,2 & 100 \\
\hline
\end{tabular}

* 5 casos de edad no precisada

A: Totalmente recuperados

B: Recuperados de una complicación de un problema básico que persiste

C: Persistencia de la patologia que motivó la hospitolización

D: Rehospitalización

E: Empeorados

F: Fallecidos

\section{Comentario}

La capacidad resolutiva que tienen los hospitales respecto a los niños y adolescentes internados puede calificarse de alta. En efecto, $70 \%$ de éxito a 30 días después del alta, entendiendo por tal la recuperacion completa y vuelta al estado de sano, es satisfactorio. El resultado global de los tres hospitales estudiados, que incluye adultos y niños, muestra $60 \%$ de recuperación completa ${ }^{6}$, proporción que se reduce a $53 \%$ en los enfermos mayores de 20 años. Paralelamente, en la atención hospitalaria infantil la tasa de letalidad encontrada es baja $(0,2 \%)$, pero en cambio se registra una proporción relativamente elevada de niños rehospitalizados $(9,6 \%)$ en el período de 30 días comprendido a partir del momento del alta.

Los mejores o peores resultados de la hospitalización dependen grandemente del tipo de pacientes que ingresa a los establecimientos, lo que a su vez deriva de las políticas vigentes de internación hospitalaria. En el caso de la hospitalización de niños y adolescentes seguidos en 
Tabla 3

Resultados a 30 dias según tipo de patologfa

\begin{tabular}{|c|c|c|c|c|c|c|c|c|}
\hline Género & \multicolumn{8}{|c|}{$R$ es ul a a d os $(\%)$} \\
\hline Respiratoria & 119 & 68.9 & 1.7 & 18.5 & 10,1 & 0,8 & - & 100 \\
\hline Perinatal & 98 & 84.7 & - & 3.1 & 12,2 & - & - & 100 \\
\hline \multicolumn{9}{|l|}{ Infecriosas } \\
\hline transmisibles & 60 & 78,2 & - & 16,7 & 1,7 & 1,7 & 1,7 & 100 \\
\hline Digestiva & 38 & 73,7 & - & 18,4 & 7,9 & - & - & 100 \\
\hline Traumatismos & 30 & 43.3 & - & 50,1 & 3,3 & 3,3 & - & 100 \\
\hline Genitourinarias & 19 & 57,9 & - & 31,6 & 10,5 & - & - & 100 \\
\hline \multicolumn{9}{|l|}{ Nerviosa y órganos } \\
\hline de sentidos & 14 & 42.9 & - & 21,4 & 35,7 & - & - & 100 \\
\hline Congénitas & 14 & 35,7 & 7.1 & 57.2 & - & - & - & 100 \\
\hline Otras causa* & 22 & 66,7 & - & 33,3 & - & - & 100 & \\
\hline Total & 414 & 70,0 & 0,7 & 18,8 & 9,5 & 0,7 & 0,2 & 100 \\
\hline
\end{tabular}

* Corresponden a enfermedades del aparato locomotor (7 casos), de la piel (4), tumores (4), circulatorias (3), endocrinas (3) y mal definida (1)
A: Totalmente recuperados
B: Recuperados de una complicación de un problema búsico que persiste
C: Persistencia de la patologta que molivó la hospilalización
D: Rehospitalización
E: Empeorados
F: Fallecidos

\section{Tabla 4}

Resultados a 30 días segûn longitud de la estada hospitalaria

\begin{tabular}{lrrrrrr}
\hline Dias & & \multicolumn{7}{c}{ Res I t ad o s (\%) } \\
de estada & n casos & \multicolumn{1}{c}{ A } & \multicolumn{1}{c}{ C } & \multicolumn{1}{c}{ D } & Otras & Total \\
\hline-1 & 124 & 62,7 & 21,3 & 11,0 & 5,0 & 100 \\
$2-5$ & 230 & 79,6 & 15,1 & 5.3 & - & 100 \\
$6-14$ & 28 & 59,8 & 33,9 & - & 6,3 & 100 \\
$15+$ & 32 & 48,6 & 12,8 & 27,7 & 10,9 & 100 \\
\hline Total & 414 & 70,0 & 18,8 & 9,5 & 1.8 & 100 \\
\hline
\end{tabular}

A: Tolalmente recuperados

C: Persistencia de la patologia que motivó la hospitalización

D: Rehospitalización

Otras: Empeoramiento y fallecimiento

este estudio, los resultados aparecen asociados al tipo de patología y a la edad de los niños. Conviene comentar los diversos resultados encontrados según longitud de la estada hospitalaria. Al igual que en el caso de los adultos ${ }^{6}$, los pacientes con estadas muy cortas tienen peor resultado que aquellos con estadas algo mayores. En el otro extremo, con alguna lógica, se advierte que prolongaciones grandes de Ia estada no generan necesariamente buenos resultados.

En los niños y adolescentes sometidos a métodos quirúrgicos, los resultados son inferiores a los observados en aquellos que recibieron tratamientos no quirúrgicos. La diferencia, que es estadísticamente significativa, deriva de una mayor proporción de patologías crónicas -de peor evolución- en el grupo quirúrgico, en comparación con una proporción alta de enfermedades agudas recuperadas en el grupo sometido a tratamientos médicos.

El cumplimiento de las indicaciones dadas en el momento del alta aparece como un factor de importancia en los resultados observados. Ello es concordante con lo observado en los pacientes infantiles y adolescentes ambulatorios en nuestra experiencia?. La importancia de esta conducta no se registra en los pacientes hospitalizados adultos ${ }^{6}$.

Resultan sorpresivos los mejores resultados observados en pacientes del subsistema FONASA de atención médica, comparados con otros subsistemas previsionales. En la atención ambulatoria estudiada por nosotros ${ }^{7}$, los mejores resultados se asocian al nivel socioeconómico 


\section{Tabla 5}

Resultados según otras variables

\begin{tabular}{lcc}
\hline Factores anglizados & n cesos & $\begin{array}{c}\text { \% con completa } \\
\text { recuperación }\end{array}$ \\
\hline Tipo de tratamiento & & \\
$\quad$ Médico & 268 & 79,8 \\
$\quad$ Quirúrgico & 146 & 52,0 \\
Condición al alta & & \\
$\quad$ Recupemado & 382 & 73,8 \\
$\quad$ Jgual o empeorado & 22 & 23,9 \\
Cumplituiento de indicaciones & & \\
$\quad$ posalla & & \\
Completo & 389 & 70,6 \\
$\quad$ Pancial y no cumplimiento & 25 & 57,4 \\
Tipo de previsión & & \\
$\quad$ FONASA & 286 & 79,8 \\
$\quad$ ISAPRES & 25 & 59,8 \\
$\quad$ Otras & 96 & 47,9 \\
\hline
\end{tabular}

alto. La explicación de los hallazgos en pacientes hospitalizados deriva del hecho que los tipos de patología por los que se hospitalizan niños y adolescentes beneficiarios de FONASA difieren significativamente $(\mathrm{p}-0,05)$ de los diagnósticos de los otros grupos. En estos se registran menores proporciones de palologias de mejor resultado como las respiratorias, y mayores proporciones de enfermedades digestivas, urogenitales 0 traumatismos, grupos de patología con resultados inferiores.

Un adecuado funcionamiento hospitalario representa un elemento clave en la atención médica chilena. Conviene recordar que la dotación total de camas públicas y privadas se incrementa con una velocidad inferior al crecimiento poblacional del pais y que no existen planes en los subsectores de atención para modificar esta tendencia. Por otra parte, los cambios demográfi$\cos$ y tecnológicos de la medicina generan nuevas necesidades a resolver. De lo anterior se desprende que en el futuro los hospitales serán requeridos en forma diversa a la actual ${ }^{8}$. generándose presiones para conocer cada vez con mayor precision la eficacia real de los establecimientos y el costo-efectividad de las acciones realizadas.

\section{Referencias}

1. Medina E, Kaempffer AM: Los hospitales chilenos: dotación y productividad de los sectores público y privado. Rev Med Chile 1992; 120: 334-34 J.

2. Kaempffer AM, Medina E: Hospitalización infantil en Chile: situación actual y perspectivas. Rev Chil Pediatr 1992; 63: 110-117.

3. Medina E, Kuempffer AM: Hospitalizaciones en Chile: análisis crítico. Rev Med Chile 1992; 120: 702-708.

4. Medina $E$ : Indicadores de salud y gestion en salud. Rey Admin Salud 1989; 7: 20-25.

5. OPS/OMS. CIE 10 Clasificación Estadística Internacional de Enfermedades y Problemas Relacionados con la Salud. Décima Revisión. Publ Cientif OPS N ${ }^{\circ}$ 554, 1995 Washington, EE.UU.

6. Kuempffer AM, Medina E Cornejo E, Hernández E: Resultados alejados de la atención médica en Santiago. Rev Med Chile 1998; 163-167.

7. Medina E. Kaempffer AM, Cornejo E. Hernóndez E: Evaluación de resultados de la atención médica ambulatoria de nifios y adolescentes. Rev Chil Pediatría 1998: 69: 168.172.

8. Medina E. Kaempffer AM: La salud en Chile: reflexiones para el futuro. Rev Med Chile 1990; I 18: 1165. 1172 . 